\title{
Sur la règle de reconnaissance
}

Conferência proferida no Colóquio Franco-Brasileiro "O Estado. Filosofia Moral e Política", realizado na Ecole Normale Supérieure de Fontenay/St-Cloud em novembro de 1990. Publicado anteriormente nos "Cahiers de Fontenay" n⿳0 67/68 (setembro de 1992)

\section{Paulo Faria}

Professor do Departamento de Filosofia do

Instituto de Filosofia e Ciências Humanas da UFRGS

\section{Abstract}

Le scepticisme dans le domaine pratique re pute indécidable toute question touchant, soit l'existence, soit le contenu, d'une règle ou d'un ensemble de regles. Dans The Concept of Law, H.L.A. Hart a essayé de démontrer l'insoutenabilité de cette vue, moyennant la caractérisation de l'usage propre des propositions déontiques. Un examen attentif de la doctrine de Hart rend explicite le prix à payer (dans le domaine prati que non moins que dans celui de la connaissance) pour se débarrasser du scepticisme; une moralité politique est alors dégagée.

Scepticism in the practical realm deems undecidable any question concerned with either the existence or the content of a rule or a series of rules. In The Concept of Law, H.L.A. Hart tried to demonstrate the indefensibility of this view, by means of characterising the proper usage of deontic propositions. A careful examination of Hart's doctrine makes clear the price that must be paid (in the practical sphere no less than in that of knowledge) if scepticism is to be got rid of. A political moral is offenal.

Der Skeptizismus im praktischen Bereich schätzt jede Frage, die entweder von der Existenz oder des Inhalts einer Regel oder eines Ensembles von Regeln behandelt, als unentscheidbar ein. In The Concept of Law, bat H.L.A. Hart die Unbaltbarkeit einer solchen Lebre mittels einer Kennzeichnung eines eigenständigen Gebrauchs von deontischen Aussagen zu beweisen versucht. Eine eingehende Prüfung der Doctrin Harts macht den Preis deutlich, der (im praktischen Bereich nicht weniger als im Bereich der Er- kenntnis) zu zablen ist, wenn mann sich von dem Skeptizismus befreit; eine politische Moralität ist nämlich damit freigesetzt.

El escepticismo en el dominio práctico considera indecidible toda cuestión que afecta sea la 'existencia', sea el 'contenido', de una regla o de un conjunto de reglas. En 'The Concept of law', H.L.A. Hart trata de demostrar la insostenibilidad de este punto de vista, mediante la caracterización del uso proprio de proposiciones deónticas. Un examen detenido de la doctrina de Hart hace explícito el precio a pagar (en el campo práctico y también en el del conocimiento) para deshacerse del escepticismo. Una lección política se desprende de abí.

On se souviendra de ce passage remarquable de la Préface à la Première Edition de la Critique de la Raison Pure, où Kant appelait les sceptiques "une espèce de nomades, qui ont en horreur tout établissement stable sur le sol" et qui, profitant des "guerres intestines" qui déchiraient le royaume de la Métaphysique, "rompaient de temps en temps le lien social" (Kant, 1781, A IX) Cette métaphore politique n'empêchait pas, le moins du monde, le compliment adressé à ces nomades, dont les activités subversives faisaient preuve d'une "façon de penser qui va au fond des choses" (A XI) - compliment auquel faisait écho, dans la Dialectique Transcendantale, le plaidoyer pour une "méthode sceptique" (A 423/B 451) consti- tuant un moment essentiel de la philosophie critique elle-même.

Or, il se trouve peut-être que ce jugement favorable s'appuie, au fond, sur la certitude de ce que, quoi qu'ils pensent, nomades et sédentaires sont citoyens d'au moins une patrie commune - à savoir le discours. La citoyenneté du nomade serait alors trahie, pourvu que, tout simplement, il prenne la parole: pour affirmer, nier ou douter, peu importe. Toute menace d'anarchie serait défaite du moment où l'on reconnaîtrait la prétention de sens dont la voix du sceptique, pas moins que n'importe quelle autre, est porteuse ${ }^{1}$

Os est en droit de se demander ce que cette idée pourrait apporter à l'interrogation philosophique sur les fondements de l'ordre politique - ou plutôt sur l'idée même d'un fondement, au sens philosophique, du politique. C'est la question que je vais tâcher d'éclaircir, sinon de résoudre, par la voie d'une réflexion sur le concept, introduit par H.L.A. Hart dans The Concept of Law (Hart 1961), d'une "règle de reconnaissance". Cette discussion trouve son point de départ dans une considération plutôt anodine: à savoir que "reconnaissance", "reconnaître" sont des concepts épistémiques - et, qui plus est, le concept d'une règle de reconnaissance est explicitement introduit par Hart pour rendre compte d'un problème épistémique: celui de l'incertitude sur l'existence ou le contenu d'une règle ou d'un système de règles.

J'aimerais placer cette discussion sous l'enseigne de Franz Kafka, qui, presque un siècle et demi après la parution de la Critique de la Raison Pure, opposait à l'optimisme kantien qu'on vient d'évoquer un jugement foncièrement négatif. Voilà ce que dit, dans un document plein d'intérêt, un commerçant angoissé:

"On ne peut pas parler avec les nomades. Ils ne connaissent pas notre langue, c'est même à peine s'ils en ont une pour eux. Pour se comprendre entre eux, ils crient comme les choucas. On ne cesse d'entendre ces cris (de choucas). Nos moeurs et nos coutumes leur sont aussi incompréhensibles qu'indifférentes. Aussi ne veulent-ils rien savoir de ce qu'on cherche à leur dire par gestes. On a beau se décrocher les mâchoires et se tordre les poignets, ils ne vous comprennent quand même pas, ils ne vous comprendront jamais". (Kafka, 1917, 489).

J'aurai l'occasion de revenir à ce texte remarquable. Pour le moment, tâchons de cerner notre problème, tel qu'il se pose pour Hart dans son ouvrage de 1961.

Le concept de reconnaissance est introduit par Hart, dans The Concept of Law, moyennat la fiction d'un système social gouverné exclusivement par des règles imposant des devoirs ${ }^{2}$ - soit ce que Hart appelle des règles "primaires". Cela équivaut à la fiction d'une société "sans législature, tribunaux ou autorités d'aucune sorte" (Hart 1961, 89) - étant donnée l'absence de règles "secondaires" octroyant des pouvoirs. La question de savoir si de telles "sociétés sans Etat" existent ou ont existé empiriquement est sans importance. Le problème à discuter est conceptuel, et peut être caractérisé comme un problème de décision: il s'agit de spécifier des critères d'attribution de valeur de vérité à des propositions sur l'existence ou le contenu des règles primaires. Ces critères, s'il y en avait, seraient, eux aussi, des règles - à distinguer, cependant, en tant que règles de deuxième ordre, des règles primaires dont l'existence ou le contenu seraient déterminés par leur moyen. La difficulté tient, précisément, à ce que le système n'a, par définition, aucune règle de deuxième ordre:

"Alors, si des doutes sont levés sur la question de savoir quelles sont les règles, ou quelle est la portée exacte d'une certaine règle donnée, il n'y aura pas de procédé pour trancher la question, soit par référence à un texte doué d'autorité, soit à un fonctionnaire dont les déclarations à ce propos seraient douées d'autorité. Car, évidemment, un tel procédé, et la reconnaissance, soit d'un texte, soit de personnes, doues d'autorité, suppose l'existence de règles d'un type différent des règles d'obligation qui, ex bypothesi, sont tout ce que le groupe possède". (Hart, 1961, 90) 
Le remède à cette déficience doit être cherché dans l'introduction d'une règle de reconnaissance, laquelle aura à spécifier "un ou plusieurs aspects dont la possession par une règle quelconque constituera une indication conclusive de ce qu'il s'agit d'une règle du groupe, à être supportée par la pression sociale qu'il exerce" (Hart, 1961, 92)

La règle de reconnaissance est ainsi la forme la plus élémentaire de ce que Hart appelle les règles secondaires - des règles fournissant des critères d'individuation, d'introduction, de supression et d'application des règles primaires d'obligation. Ces règles de deuxième ordre fournissent aussi bien les critères requis d'existence et d'identité pou un système juridique, en spécifiant des procédés d'attribuiton de valeur de vérité à des propositions sur l'appartenance au système des règles primaires. La distinction entre les deux ordres de règles fonde finalement l'élucidation du concept même de droit: l droit se laisse caractériser comme l'union des règles primaires d'obligation avec de telles règles secondaires (Hart, 1961, 91)

On remarquera qu'avec l'introduction du concept d'une telle règle secondaire c'est le mot "règle" qui acquiert en fait un sens nouveau. La règle de reconnaissance est, en effet, vide de contenu "prescriptif"; elle ressemble plutôt à une définition, ou à un ensemble de définitions, qu'à une proposition, ou un ensemble de propositions déotinques (Bulygin, 1976). Cette différence est d'ailleurs soulignée par Hart lui-même, dans le contexte d'un argument en faveur de la nature catégorielle de la distinction entre règles primaires et secondaires. L'examen de la structure de certains jeux complexes (comme le football) ou de celle de certaines institutions morales (comme la promesse) mène au même résultat:

"Dans la morale, les règles vagues qui déterminent si une personne a fait une promesse sérieuse confèrent des pouvoirs limités de législation morale à des individus, et doivent ainsi être distinguées des règles qui imposent des devoirs in invitum (...). Les règles de tout jeu complexe peuvent aussi être étudiées profitablement de ce point de 106 vue. Certaines règles (analogues à celles du droit pénal) prohibent, sous peine, certains types de comportement, p. ex. la faute ou l'irrespect à l'égard de l'arbitre. D'autres définissent la juridiction des autorités du jeu (arbitre, arbitre de touche); d'autres encore définissent ce qui doit être fait pour obteni des points (p. ex, le but)". (Hart, 1961, 238. e souligne, $\mathrm{PF}$ )

L'accent mis sur ce trait "définitionnel" des règles secondaires ne saurait pourtant couvrir les différentes fonctions qui reviennent à ces règles de deuxième ordre. Il faut faire attention à ces différences, car c'est dans le contraste avec d'autres variétés de règles secondaires qu'est rendu manifeste le statut problématique de la règle de reconnaissance - et, par là-même, l'admissibilité de l'argument, que je veux proposer, contre l'idée même d'un jugement conclusif de reconnaissance.

Hart est prudent à ce propos. The Concept of Law distingue trois variétés fondamentales de règles secondaires, introduites successivement à partir de l'examen de la fiction méthodologique d'un système social 'primitif', c'est-à-dire d'un système réglé exclusivement par des règles primaires d'obligation. A l'incertitude sur l'existence ou le contenu des règles primaires correspond, nous l'avons vu, le remède introduit avec la règle de reconnaissance. De manière analogue, deux autres espèces de règles secondaires sont introduites comme des correctifs à deux autres déficiences du système primitif. Ainsi chaque type de règle secondaire est conçu comme la solution d'un probleme. On peut s'attendre à ce qu'une caractérisation correcte de la nature de chaque problème fournisse un fil conducteur adéquat à une catégorisation préliminaire des règles secondaires.

Bien sûr, le système primitif n'est pas seulement incertain. Une deuxième source de difficultés se trouve dans son caractère statique:

"Le seul mode de changement de règles connu d'une telle société serait le lent processus de croissance, par lequel des cours d'action au début regardés comme faculta- tifs deviennent d'abord habituels ou usuels, et puis obligatoires, et le processus réciproque d'abandon quand des déviations, au début sévèrement traitées, sont d'abord tolérées et après passent inaperçues. Il n'y aura pas moyen, dans une telle société, d'adapter délibérément les règles à des circonstances modifiées, soit par l'élimination des vieilles règles, soit par l'introduction de nouvelles; car, encore une fois, la possibilité de le faire présuppose l'existence de règles d'un type différent des règles primaires d'obligation, qui sont les seules que la société possède". (Hart, 1961, 90)

Le remède à ce deuxième défaut - le premier étant l'incertitude à laquelle répond la règle de reconnaissance - est dans l'introduction de ce que Hart appelle des règles $d e$ changement: "La forme la plus simple d'une telle règle est celle qui confère à un individu ou à un corps de personnes le pouvoir d'introduire de nouvelles règles primaires pour la conduite de la vie du groupe, ou d'une classe quelconque à l'intérieur de ce groupe et d'éliminer de vieilles règles (Hart, 1961, 93). Ces règles de changement caractérisen un groupe de concepts juridiques, tels ceux d'un législateur ou d'une autorité administrative, comme ceux subordonnés à celui de l'autonomie privée", telle la capacité de conclure un contrat, de se marier ou de tester.

Il y a un rapport important entre ces règles de changement et la règle de reconnaissance: si un système juridique a des règles de changement, sa règle de reconnaissancè contiendra, nécessairement, une référence à des actes de changement (actes d'introduction ou de suppression) de règles primaires, et comptera les critères d'identite pour ces actes parmi les critères de détermination de l'existence des règles primaires (Hart, 1961, 93).

Le troisième défaut du régime des règles primaires est l'inefficacité de la "diffuse pression sociale" (Hart, 1961, 91) qui maintien les règles en vigueur. La controverse sur la question de savoir si une règle particulière a été violée ou non peut se poursuivre in définiment, étant donné que le système n'o- ctroie à aucun individu ou corps d'individus l'autorité pour la trancher d'une façon conclusive. A cette déficience est évidemment lié le fait, non moins indésirable, que les sanctions correspondantes à la violation des règles ne sont administrées par aucune institution spéciale, et doivent donc se résoudre en des actes désordonnés de vengeance, laissés à l'initiative des offensés ou du groupe comme un tout.

Le remède à ce troisième défaut est l'introduction de règles de juridiction octroyant à quelques individus ou groupes le pouvoir de décider avec autorité la question de savoir si, dans une occasion particulière, telle ou telle règle primaire a été violée (Hart, 1961, 94). La forme minime de juridiction est constituée par une telle décision, mais les règles de ce type contiendront, normalement, des spécifications sur le procédé juridictionnel (des règles de procédure). Comme les règles de changement, les règles de juridiction définissent aussi un groupe de concepts juridiques: dans ce cas, ceux de juge, jugement, tribunal ou procès. Comme les autres règles secondaires, les règles de juridiction n'imposent pas d'obligation: leur fonction essentielle est celle d'octroyer des pouvoirs à quelques individus ou groupes, et un statut particulier aux déclarations juridictionnelles sur l'observance ou la violation des obligations.

Dans ce cas aussi, le rapport avec la règle de reconnaissance est étroit, dans la mesure où, si un systéme juridique contient des règles de juridiction, sa règle de reconnaissance contiendra, nécessairement, une référence à des actes de juridiction, et comptera les critères 'd'identité pour ces actes parmi les critères de détermination du contenu des règles primaires. Un autre rapport, plus complexe et philosophiquement plus instructif, entre les règles de juridiction et de reconnaissance sera indiqué tout à l'heure.

Pour le moment, restons sur la comparaison suggérée entre les problemes dont la solution est fournie par l'introduction de chacun des trois types de règles secondaires.

Le contraste le plus évident est, ici, celui qui existe entre le problème de l'incertitude 
des règles primaires, dont le critère de solution est fourni par la règle de reconnaissance, et celui de son caractère statique, dont le critère de solution est fourni par les règles de changement. Car, tandis que le premie se présente comme un problème épistémique. le deuxième a l'allure d'un problème éminemment pratique: dans le premier cas, il y a quelque chose qu'on ne peut pas savoir (s'il existe vraiment une règle primaire, ou quelle est la portée d'une règle censée exister); dans le deuxième cas, la difficulté tient à ce qu'il y a quelque chose qu'on ne peut pas faire (changer les règles primaires). Quand la comparaison est étendue aux règles de juridiction, cependant, la situation se complique. L'inefficacité de la "diffuse pression sociale" chargée d'assurer l'observance des règles primaires est, manifestement, un problème lié à l'action, pas à la connaissance. Sous cet aspect, les règles de juridiction ressemblent à celles de changement: c'est, d'ailleurs, un trait commun aux règles des deux types qu'elles octroyent des pouvoirs à certains individus ou groupes (pour introduire ou supprimer des règles primaires; pour trancher la question de son observance ou violation) et cette caractéristique est entièrement absente de la règle de reconnaissance. Mais, comme nous l'avons u, ce que Hart entend par inefficacité se manifeste, primairement, comme indécidabilité de la controverse sur l'observance ou la violation des règles primaires. Corrélativement, la forme minime de juridiction est caractérisée comme une déclaration, qui tranche une question cognitive: tel ou tel cours d'action est-il ou n'est-il pas en accord avec telle règle primaire? Il s'agit, au moins "prima facie", de quelque chose que l'on veut savoir, et le statu particulier accordé aux déclarations juridictionnelles (consistant, pour l'essentiel, dans le caractère conclusif qu'a un certain moment - usuellement défini par les règles de procédure - elles acquièrent) ne modifie pas la force déclaratoir qui est essentielle à la forme minime de juridiction (encore qu'a cette force déclaratoire vienne s'ajouter une force prescriptive, condamnatoire ou autre).

108
C'est précisément ce trait caractéristique (et l'aspect épistémique correspondant du problème de l'inefficacité) qui complique énormément le rapport entre les règles de juridiction et la règle de reconnaissance. Car, si au moins une partie de la fonction des premières consiste à déterminer la portée des règles primaires (en déclarant, avec autorité, si une certaine règle primaire a été violée ou pas), il semble inévitable que les règles de juridiction déterminent, au moins partiellement, le contenu de la régle de reconnaissance elle-même:

"En fait, un système qui a des règles de juridiction d'un certain type est aussi compromis avec une règle de reconnaissance d'un type élémentaire et imparfait. Cela arrive parce que, si les tribunaux sont investis de pouvoir pour donner des déterminations autorisées du fait qu'une règle a été violée, il est inévitable qu'elles soient prises pour des déterminations autorisées concernan quoi ou quelles sont les règles. Ainsi la règle qui confère juridiction sera anssi une règle de reconnaissance identifiant les jugements des tribunaux, et ces jugements deviendront des "sources" de droit". (Hart, 1961, 94-95)

Or, il n'est pas évident que cela ne conduise pas à une forme vicieuse de circularité: car, si les règles de juridiction doivent être, de la façon indiquée, des règles de reconnaissance, aucun argument a priori ne pourrait exclure (et, en fait, ajouterais-je, aucun système juridique n'exclut) du domaine de la juridiction les controverses sur la portée des règles secondaires.

C'est bien là que, dans l'ordre des raisons, surgit pour Hart ce qu'il appelle le scepticisme sur les règles ("rule-scepticism"), dont l'expression mûre est le soi-disant "réalisme juridique" scandinavien. Et c'est là aussi ce qui mène Hart à reposer son problème épistémique du départ comme un problème de décision de deuxième ordre, qu'il appelle maintenant "l'incertitude de la règle de reconnaissance" (Hart, 1961, 144-150). L'analogie entre cette récurrence du problème de l'incertitude et la structure d'un argument classique du scepticisme épistémique n'échappera pas à quiconque fera l'exercice sommaire de substituer par "règle de reconnaissance" le mot "critère" dans ce passage des Hypotyposes Pyrrboniennes:

"D'ailleurs, pour trancher le désaccord né à propos du critère, il nous faut avoir un critère reconnu, par le moyen duquel nous pourrions juger, et pour avoir un critère reconnu, il faut d'abord trancher le désaccord sur le critère. Nous tombons ainsi dans le mode du cercle vicieux, et il devient impossible de découvrir le critère, puisque nous ne les laissons pas choisir un critère par hypothèse, et que s'ils veulent juger un critère par un autre, nous les poussons à la régression à l'infini". (Sextus Empiricus, 1948, 218)

Le juriste danois Alf Ross, "rule-sceptic" $s^{\prime}$ 'il y en a, remarquait (dans son compte-ren$\mathrm{du}$ de The Concept of Law) que sous la rubrique "règle de reconnaissance", Hart s'occupe de ce qu'on appelle usuellement "les sources du droit" (Ross, 1962, 356). Remarque importante, s'il est vrai que, dans ses diverses variantes, le positivisme juridique s'est caractérisé par la thèse selon laquelle "le droit a des sources sociales, c'est-à-dire que le contenu et l'existence du droit peuvent être déterminés par rapport à des faits sociaux et sans aucun recours à des considérations morales" ( $\operatorname{Raz}, 1979,53)$.

Le problème évident de cette thèse est que, en faisant dépendre la validité d'une règle du fait qui, chaque fois, en constitue la source, elle tend à laisser soupçonner que parler de validité, et même de règle, n'est qu'un redoublement inutile, sinon trompeur, d'entités.

La suggestion (ou la tentation) qui s'insinue est alors celle d'éliminer, en faveur d'un langage purement "factuel", tout discours sur droits et obligations, pouvoirs et responsabilités. Pratiquement, cela reviendrait à introduire des postalats de réduction, au moyen desquels toute proposition en langage normatif se laisserait traduire dans sa contrepartie "factuelle". Voilà bien ce qu'il faudrait entendre, selon Karl Olivecrona, par la devise "law as fact": "Ce titre dit ce que j'envisage: encadrer les phénomènes complexes couverts par le mot droit dans le monde spatio-temporel" (Olivecrona, 1971, VII) ${ }^{3}$.

Une bonne partie de l'histoire du positivisme juridique, de Bentham et Austin aux Réalistes Américains jusqu’à Ross et Olivecrona, se laisse lire, en effet, comme un abandon progressif de l'idée même de la normativité du droit. C'est ainsi que des positivistes sont arrivés à dire que des propositions sur des droits, des devoirs ou des responsabilités sont équivalentes ${ }^{4}$ à des propositions sur ce que des gens déterminés ont commandé, ou voulu, ou sur les chances qu'un homme soit exposé à un certain type de violence, ou sur la probabilité que les tribunaux profèrent certaines décisions.

Voilà donc ce qui, mené jusqu'au bout, pourrait bien constituer un vrai "scepticisme sur les règles". Il va sans dire que, dans la perspective d'un tel scepticisme, il ne pourrait y avoir de place pour des questions telles que l'autorité de la loi ou l'obligation politique. Ces questions censées être indécidables doivent également trouver leurs contreparties factuelles, qui feront alors l'objet des recherches d'un Roscoe Pound sur le "contrôle social" ou d'un Alf Ross sur la "politique juridique".

Eh bien, il s'agit de savoir si c'est vraiment à une "élimination du normatif" que l'on peut s'attendre par cette voie. C'est donc, en un certain sens, l'intelligibilité du scepticisme sur les règles qui sera en cause.

A mon avis, ce qui rend intéressante la stratégie de Hart est le fait qu'il cherche, comme il se doit d'ailleurs, à "battre le sceptique sur son propre terrain". Ce sera à partir de l'affirmation, et non pas du refus, de la thèse positiviste des sources sociales du droit qu'il faudra conclure, le cas échéant, à l'intenabilité du scepticisme.

Pour voir comment cela arrive, laissezmoi revenir à ce texte de Kafka que je vous ai présenté au début de cet exposé. Nous y avons vu comment le narrateur arrivait à conclure, du fait qu'"on ne peut pas parler avec les nomades", que ce sont ce qu'il appelle "nos moeurs et nos coutumes" qui leur sont "aussi incompréhensibles qu'indifférentes". Là-dessus, rien à faire: ils "ne veu- 
lent rien savoir de ce qu'on cherche à leur dire par gestes"; décrochez vos mâchoires, tordez vos poignets, ils ne vous comprennent quand même pas:

"Il leur arrive souvent de faire des grimaces; on voit alors le blanc de leurs yeux se retourner et l'écune mousser sur leurs lèvres, mais ce n'est ni pour exprimer quoi que ce soit ni pour faire peur; ils le font simplement parce que c'est dans leurs moeurs". (Kafka, 1917, 489)

Une partie au moins de la force de ce récit provient sans doute de ce que, en le lisant, nous nous plaçons sans équivoque de ce côté-ci: celui des habitants perplexes de ce pays envahi. Nous reconnaissons dans ce récit des institutions qui nous sont familières: le commerce, l'armée, le gouvernement. Nous comprenons ce que le narrateur veut dire lorsqu'il regrette le fait "que nous ayons été bien négligents dans la défense de notre patrie" (Kafka, 1917, 488).

On ne saurait trop insister, toutefois, sur le fait que l'auteur de ce récit ne se borne pas à prendre acte de son étonnement devant ces barbares qu'il n'arrive pas à comprendre. Il enregistre aussi bien sa certitude de ce qu'en revanche "nos moeurs et nos coutumes leur sont aussi incompréhensibles qu'indifférentes". Ce qui, d'ailleurs, n'empêche pas la prudence de la réserve: "c'est même à peine" si les nomades ont une langue pour eux: on nous informe, en tout cas, que "pour se comprendre entre eux, ils crient comme les choucas".

On est donc en droit d'imaginer que quelqu'un parmi eux entreprenne la tâche de décrire, dans sa langue de choucas, notre forme de vie. Voilà le marchand de tissus qui reçoit son client: et l'observateur nomade d'enregistrer, minutieusement, les gestes et mouvements de ces agents, le drap déployé sur le comptoir, le rite qu'il a maintes fois témoigné: avant de tailler le drap, l'homme derrière le comptoir le touche, une ou plusieurs fois, avec un bâton; seulement après cette cérémonie, il le taille, et reçoit du visiteur une quantité de petites pièces métalliques. Parfois le visiteur reçoit aussi ces métaux, avec le drap, et les emporte avec 110 soi, en sortant de la maison; mais cela n'arrive pas toujours. Les nomades ont des théories diverses sur cet événement quotidien: presque toutes supposent un rapport entre les métaux et les pouvoirs sacrés du bâton; c'est là une conjecture raisonnable, car la quantité de métaux semble être proportionnelle au nombre de touches de bâton. Mais ça ne suffit pas à expliquer pourquoi certains parmi les visiteurs reçoivent des métaux et d'autres pas. Tous les apportent et tous reçoivent du drap, c'est certain; le reste est encore matière à controverse.

Il est aisé de voir que ce récit n'est pas loin des spéculations de Clefrin Frelock et Marsi Frelock, chargés par le Roi de Lilliput de faire l'inventaire des biens personnels de Gulliver, à propos de ce disque, moitié argent et moitié une sorte de métal transparent, trouvé au bout d'une chaîne, dans le gilet de l'Homme-Montagne: merveilleux engin qui "faisait un bruit incessant comme celui d'un moulin à l'eau" et que les commissaires concluaient être le Dieu que la créature adorait, car n'avait-il pas lui-même assuré "que rarement il faisait quoi que ce soit sans le consulter?". En fait, "il l'a appelé son oracle, et il a dit qu'il indiquait le temps pour chaque action de sa vie" (Swift, 1726: 18; cf. MacCormick, 1978, 275).

Dans les deux histoires, ce qu'il peut y avoir d'insolite ou de dérisoire dépend également, encore une fois, d'un contraste fondamental entre notre point de vue et celui de ces observateurs perplexes. Ce contraste se manifeste précisément dans le fait que nous savons quelque chose que ces observateurs ne savent pas: nous le savons parce que nous reconnaissons dans ces objets un instrument de mesure, des monnaies, une montre; nous les reconnaissons parce que nous savons employer ces objets; parce que nous prenons des mesures, nous achetons et nous vendons, et nous comptons les heures. En réalisant ces activités, nous faisons plus que toucher un morceau d'étoffe avec un bâton regarder un disque vitré ou manier des pièces métalliques: nous faisons usage de règles, dans lesquelles sont cristallisés les con- ventions métriques, le système monétaire, l'institution de la propriété.

Cette opposition entre les deux points de vue, baptisés respectivement l'aspect interne et l'aspect externe des règles, est la pierre angulaire de la conception du droit de Hart, et assurément la principale nouveaute que son livre a introduit dans la philosophie du droit. C'est, en effet, par un traitement adéquat des rapports entre ces deux aspects des règles que Hart se propose $d$ développer une théorie de la reconnaissan ce, laquelle aura à rendre compte des conditions d'attribution de valeur de vérité à des assertions sur des règles ou des systèmes de règles, tout en évitant et le Scylla du normativisme (dans ses diverses variantes, des doctrines classiques du droit naturel la théorie pure du droit), et le Charybde du "réalisme", c'est-à-dire de ce que Hart appelle, justement, "rule-scepticism" (Hart, 1961, 132-137). La solution proposée équivaut, comme l'annonce Hart lui-même (dans la Préface de 1983 à ses Essays in Jurisprudence and Pbilosophy), à l'introduction, dans la théorie du droit, d'une

"méthode herméneutique, impliquant la représentation du comportement gouverné par des règles tel qu'il apparaît à ses participants, qui le voient comme s'accordant ou ne s'acordant pas à des modèles partagés". (Hart, 1983, 13)

La distinction entre l'aspect interne et l'aspect externe des règles peut être éclairée (comme elle l'est, d'ailleurs, par Hart) par celle entre habitudes (régularités de conduite) et comportements gouvernés par des règles, telle qu'elle est présentée par Winch dasns The Idea of a Social Science (Winch, 1958, 57-65).

Ce qui rend facile la confusion entre habitude et comportement gouverné par des règles est le fait très évident que, dans les deux cas, le comportement en question "doit être général, bien que non invariable; cela veut dire qu'il est répété chaque fois que l'occasion se présente pour la majeure partie du groupe" (Hart, 1961, 54). D'où la phrase: "Ils font ça comme une règle " ("They do it as a rule"). En somme, et habi- tudes et comportements réglés sont également "réguliers".

En dépit de cette ressemblance, pourtant, au moins trois différences importantes sont à signaler:

1.- Pour qu'il y ait une habitude, il suffit que les cours d'action des individus soient convergents. Dans ce cas, la déviation n'a pas à donner lieu à la critique, à la réprobation, ou à la pression pour la conformité. En revanche:

"quand il y a une règle, les déviations sont généralement regardées comme des lapsus ou des fautes exposées à la critique, et des menaces de déviation font face à la pression pour la conformité, bien que les formes de-critique et de pression soient différentes pour des types différents de régle". (Hart, 1961, 54).

2.- Là où il y a des règles, et pas seulement de simples régularités de conduite, non seulement il y a critique, mais la critique est légitimée par rémission aux règles. En d'autres mots, les règles constituent une bonne raison pour les critiques et les justifications de conduite.

3.- Quand une habitude est généralisée dans un groupe, cette généralité est simplement un fait à propos du comportement observable du groupe, ou de sa majeure partie:

"Pour qu'il y ait une telle habitude, aucun membre du groupe n'a à penser sur le comportement général, ou même à savoir que ce comportement est général; moins encore doivent-ils s'occuper de l'apprendre ou avoir de dessein de le maintenir". (Ibid.)

En revanche, si une règle sociale doit exister, au moins quelques-uns doivent considérer le comportement en question comme un modèle qui doit être suivi par le groupe comme un tout (Ibid.). Cette attitude particulière constitue l'aspect interne des règles.

Les jouers d'un jeu n'ont pas seulement des habitudes semblables (disons, une certaine façon de déplacer la reine sur l'échiquier), des habitudes qu'un observateur externe peut enregistrer; ils ont "une attitude critique réflexive envers ce modèle de comportement: ils le considèrent comme un éta- 
lon pour tous ceux qui jouent le jeu" (Hart, 1961, 55).

Jusqu'ici, nous avons parlé de l'opposition entre les deux aspects des règles comme s'il agissait, pour l'essentiel, d'une opposition entre deux "points de vue". Hart luimême utilise souvent ce jargon: "externe" est, par exemple, le "point de vue prédictif" de l'observateur qui enregistre des régularités de comportement (avec ou sans allusion au fait que ce comportement, et les réactions observables, des membres de la communauté, sont vus par eux comme s'accordant ou ne s'accordant pas avec des règles); "interne" est alors le point de vue des membres de la communauté qui "font usage de règles pour justifier, exiger ou reprouver des actions" (Hart, 1961, 244). Cette manière de présenter les choses a, néanmoins, l'incovénient de suggérer que l'opposition interne/externe pourrait se ramener à une question d'attitudes" (encore un mot fourvoyant employé par Hart), voire de "conceptions" ou d'appartenances", idéologiques ou autres, alors que ce qui est primairement (et, comme nous le.verrons, décisivement) visé est une distinction entre deux types d'énoncés, c'est-à-dire entre deux usages du langage.

Pour tirer cela au clair, considérons un peu les choses du côté du comportement des agents, lorsqu'il s'agit de comportement gouverné par des règles (par opposition à la simple habitude). Il faudra, d'après ce que nous venons de voir, introduire une distinction entre l'observance (ou conformité avec la règle) et l'acceptation (ou usage de la règle comme un étalon de comportement). L'une et l'autre sont requises pour que l'on puisse parler de comportement gouverné par des règles, et, par là même, maintenir la distinction entre l'aspect interne et l'aspect externe des règles. Il ne faut pas, néanmoins, les confondre: nous le verrons, c'est finalement cette distinction qui soutient le poids de la théorie de la reconnaissance, et, par cette voie, du réglement de comptes de Hart avec son sceptique.

Grammaticalement, qu'est-ce que l'observance? C'est la réalisation du cours d'action exigé ou permis par la règle. Il y a entre 112 règle et observance un rapport interne, en ce sens que, comme le soulignent Baker et Hacker, "comprendre une règle est savoir quels actes seront en accord avec elle, tout comme comprendre une assertion est savoir ce qui serait le cas si elle était vraie" (Baker \& Hacker, 1984, XIII). Ce rapport interne se laisse reconnaître dans la dépendance logi que entre l'énoncé de la règle et la description du cours d'action qui, sous cette description ${ }^{6}$, lui est conforme. Ainsi la règle "Il est défendu de conduire à une vitesse supérieure à $100 \mathrm{Km} /$ heure" est observé par X si, et seulement si, $\mathrm{X}$ se garde de conduire à une vitesse supérieure à $100 \mathrm{Km} /$ heure $^{7}$

Par contre, l'acceptation peut être caractérisée comme un rapport "pragmatique" externe entre règle et action: l'acceptation est ce que Hart appele l'usage de la règle comme un étalon de conduite.

Il ne s'agit pas, il faut le souligner, d'une quelconque "attitude" psychologique envers la règle, mais de quelque chose que l'on fait: dans un sens très précis, donc, c'est toujours de comportement qu'il s'agit. C'est d'ailleurs ce qui fait que l'on puisse s'attendre à "battre le sceptique sur son terrain propre" par cette voie.

Mais s'il y a une chose telle qu'un comportement d'acceptation, qui soit comme la manifestation ouverte et publiquement observable de cette "attitude envers la règle," ce doit être autre chose que l'observance. En fait, je crois que l'exigence doit être ici encore plus stricte: comportement d'acceptation et observance doivent être logiquement indépendants, en ce sens qu'une proposition de la forme "X accepte la règle $\mathrm{R}$ " n'implique pas une autre de la forme " $\mathrm{X}$ observe la règle $\mathrm{R}$ ", ni inversement.

Soit la règle "Il est défendu de conduire à une vitesse supérieure à $100 \mathrm{Km} /$ heure". Sous quelles conditions serait-on autorisé à dire qu'un tel accepte cette règle? Tout d'abord, il ne sera pas suffisant, pas même nécessaire, que cet individu observe cette règle. Ce n'est pas le fait que les gens conduisent à des vitesses inférieures à 100 $\mathrm{Km} /$ heure que l'on pourrait prendre pour acceptation de quoi que ce soit (ils peuvent le faire, d'ailleurs, pour toutes sortes de raison: par exemple, parce que leurs voitures ne développent pas des vitesses supérieures).

Alors quoi? Selon Hart, l'acceptation se manifeste dans ce qu'"au moins quelquesuns considèrent le comportement en question comme un étalon général qui doit être suivi par le groupe comme un tout" (Hart, 196, 55; je souligne, PF). En apparence, bien sûr, cela ne ferait que déplacer la difficulté: que quelques-uns "considèrent" le comportement en question comme un étalon qui doit être suivi, n'est-ce là qu'une question de croyances, voire de sentiments ou autres "états d'esprit"8?

Manifestement ce n'est pas là l'intention de Hart, qui met en garde contre toute interprétation psychologique du concept d'acceptation (et de l'aspect interne des règles):

"L'aspect interne des règles est souvent pris à tort pour une question de "sentiments", en opposition au comportement physique publiquement observable". (1961, 56)

Cette remarque doit être prise au sérieux: elle contient, en effet, le noyau de la critique de Hart au "réalisme juridique". Cette école, comme l'écrit Hart à propos de Ross dans "Scandinavian Realism":

"prend à tort l'aspect interne des règles pour une question d'émotion, ou de "sentiment" - pour une "expérience" psychologique particulière". (Hart, 1959, 166)

Que l'acceptation ne soit pas une expérience psychologique, néanmoins, n'implique pas qu'il s'agisse de "comportement physique publiquement observable". Dans "Scandinavian Realism", Hart remarque que la question entière est mal posée quand on la réduit à la disjonction entre "statements of fact" et "expressions of feeling":

"La distinction entre l'externe et l'interne n'est pas une distinction séparant le comportement physique du sentiment, bien qu'une telle distinction puisse évidemment être faite; c'est une distinction séparant deux types radicalement différents d'assertion, pour lesquels une occasion se présente dès qu'un groupe conduit ses affaires selon des règles". (Ibid.)
Je prends cette remarque pour l'annonce, en fait, d'un résultat absolument général: l'acceptation se manifeste dans le comportement linguistique des membres de la communauté: dans le fait que certaines action sont critiquées, justifiées ou exigées par renvoi à des règles (dont l'expression linguistique constitue chaque fois la prémisse, ouverte ou tacite, d'un argument pratique). Le critère de l'acceptation est donc toujours un acte de langage. C'est dans ce sens que l'acceptation n'est pas une expérience psychologique, ni un pur comportement physique observable?.

On commence à deviner la "troisième voie" par laquelle Hart entend trancher la querelle entre normativisme et réalisme: l'acceptation est le mode d'existence des règles sociales; le jugement de reconnaissance ne renvoie donc ni au normatif "pur", comme chez Kelsen ou les doctrines du droit naturel, ni à la pure facticité, comme chez Ross ou Olivecrona. Dans un sens, c'est l'opposition même entre "fait" et "règle" qu'il faudra repenser.

Remarquons cependant qu'il y a au moins deux manières, nettement distinctes, de "prendre en compte", au niveau $d u$ discours, l'existence des règles sociales. L'usage de ces règles pour justifier, critiquer ou exiger des actions en est une: c'est ce que nous avons appelé l'acceptation. Dans ce premier cas, l'usage du langage est, dans un sens très strict, constitutif: justifier, critiquer ou exiger des actions, voilà proprement à quoi sert une règle ${ }^{10}$. Cet usage se manifeste, typiquement, dans des énoncés tels que: "Il est défendu de conduire à des vitesses supérieures à $100 \mathrm{Km} /$ heure" (dit, par exemple, par un juge en imposant une sanction). Cette même phrase, pourtant, pourrait être proférée, sans aucun "compromis" normatif, par quelqu'un qui ne ferait par là que constater le fait que, dans telle ou telle communauté, ou en accord avec telle ou telle loi, il est défendu, etc. Il ne faudrait évidemment pas dire que celui qui ferait cette constatation accepterait par là quoi que ce soit (si ce n'est, dans un sens plutôt flou d'"accepter", le fait de l'acceptation de la règle par les autres). 
Dans cet usage, la phrase: "Il est défendu... n'est pas une "proposition déontique", mais bien la description d'un fait.

Ce deuxième type d'énoncés, néanmoins, n'est pas à confondre avec les énoncés "externes", au sens de Hart, proférés par u observateur qui se bornerait par là à enregistrer des régularités de conduite. Il ne s'agi pas seulement du fait que, dans les énoncés en question, on fait usage du vocabulair normatif absent, ex bypotbesi, du discour externe. Ce qui est décisif est l'inclusion, parmi les conditions de vérité de ces énon cés, du fait de l'acceptation des règles dont i $\mathrm{y}$ est question.

Ces énoncés proférés d'un "point de vue" qu'on ne pourrait pas appeler externe, mais qu se distinguent néanmoins des énoncés internes où l'on fait usage des règles en réalisant des actes de langage tels que la censure ou la justification d'une action, je les appellerai, en reprenant le mot de Joseph Raz, énoncés détachés; et j'appellerai, par contras te, engagés les énoncés internes qui manifestent l'acceptation des règles.

Ces énoncés détachés sont ceux qui caractérisent le discours du scientiste social de Winch, ou celui du juriste kelsenien: dan les deux cas, les règles dont l'acceptation es présupposée ne sont pas employées, mais seulement mentionnées (c'est pour cela que l'acceptation appartient aux conditions de vérité des assertions que l'on fait)

Il est essentiel de remarquer qu'aucune forme verbale n'est apte à distinguer les énoncés engagés des énoncés détachés (tou comme il n'y a pas de forme verbale apte à distinguer usage et mention d'une expression linguistique ${ }^{11}$ ). C'est bien pour cette raison que la distinction proposée oppose deux types d'énoncés, et non de propositions ${ }^{12}$. Cette remarque offre, comme l'a reconnu Hart lui-même, la clé pour la doctrine critique de Kelsen sur les "propositions de devoir" (Sollsätze) de la science du droit.

Comme on le sait, Kelsen soutenait qu'une même proposition peut être utilisée dans la réalisation d'un énoncé prescriptif (par le législateur, disons), cas dans lequel elle manquera de valeur de vérité; ou dans la 114 réalisation d'un énoncé descriptif (par le juriste, notamment), cas dans lequel elle sera vraie ou fausse selon qu'une telle norme appartient effectivement ou pas au système en question:

"La différence s'exhibe en ce que les énoncés déontiques proférés par la science du droit, lesquels décrivent le droit mais n'obligent ni n'autorisent quoi que ce soit à personne, peuvent être vrais ou faux; tandis que les normes produites par l'autorité juridique, qui obligent ou autorisent les sujets du droit, ne sont ni vraies ni fausses; tout comme les faits empiriques ne sont ni vrais ni faux, mais existent ou n'existent pas, tandis que les énoncés sur ces faits peuvent être vrais ou faux". (Kelsen, 1960, 86)

Dans l'introduction de 1983 aux Essays in Jurisprudence and Philosopby, Hart reconnaît explicitement cette variété d'énconcés détachés, qu'il appelle maintenant "un troisième type d'énoncés, qu'il faut ajouter aux deux (énoncés internes et externes) que je distingue (Hart, 1983, 14). Et il remarque:

"Pour tirer tout cela au clair j'aurais dû souligner qu'à côté de la distinction entre de simples régularités de conduite et des comportements gouvernés par des règles, nous avons besoin d'une distinction entre l'acceptation des règles et la reconnaissance de son acceptation par les autres". (Ibid.)

Et de reconnaître, maintenant, que ces énoncés "détachés" étaient ce que Kelsen avait en vue lorsqu'il parlait de "Sollsätze" (Hart, 1983, 15; cf. pour le jugement antérieur de Hart sur Kelsen à ce propos, Hart, $1963,287-295)^{13}$

Nous sommes en état maintenant de résumer ce qui précède en faisant remarquer que cette prise en compte de l'acceptation de règles, donc d'un certain usage du langage, qui distingue les énoncés détachés de ceux qu'on pourrait appeler, par contraste, "radicalement externes", est justement ce qui est viśé par le concept de reconnaissance.

La clé du règlement de comptes de Hart avec le "rule-scepticism" est donc dans sa caractérisation de la nature des discours où 1 est typiquement question de droits, de devoirs, de fautes ou de responsabilités: dans son usage primaire, les propositions de droit sont déontiques ou pratiques, et non pas cognitives; elles sont employées pour exiger et justifier des actions; elles entrent donc dans le discours d'une manière totalement différent de celle qui caractérise les propositions théoriques (cognitives) (Cf. Hart, 1953, 8-12).

Le seul usage proprement cognitif de ces propositions, celui qui distingue les énoncés détachés de la théorie du droit, devra être caractérisé comme parasitaire de cet usage pratique, au sens précis où il faudra compter, parmi les conditions de vérité de compter, parmi les conditions de vérité de ces assertions détachées, l'acceptation des règles qui, nous l'avons vu, s'exh

réalisation d'énoncés engagés.

La question de savoir, maintenant, si c'est à une réfutation du "scepticisme sur les règles" que l'on a abouti ainsi reste cependan ouverte. Evidemment, tout dépend de ce que l'on veut appeler "réfutation"; mais l'njeu est loin d'être purement verbal. Tou d'abord, remarquons qu'il s'agit de savoi ce qu'est une réfutation philosophique; et ensuite, que cette question enveloppe celle qui porte, au niveau le plus général, sur l'idé même d'une preuve philosophique.

Je ne peux pas discuter ici ces questions $^{14}$. Je me bornerai à offrir quelques remarques sur la conception de justificatio qui, à mon avis, est impliquée par la démarche de $\mathrm{Hart}^{15}$.

Pour ce faire, il sera utile de revenir un moment au passage que j'ai cité des Hypot poses Pyrrboniennes, pour y souligner la presentation explicite de ce que l'on est venu à appeler, de nos jours, le "trilème de la justification": à savoir que toute justification débouche forcément soit sur l'arbitraire (ce que Sextus appelle "choiser un critère par hypothèse"), soit sur la circularité, soit sur la régression à l'infini. Etant donné ce trilème (et sauf à suspendre le jugement) il n'y aurait qu'à choisir entre une forme quelconque de "bon" arbitraire (que ce soit l'habitude, l'inclination naturelle ou la tradition), de "bonne" infinitude ou de "bonne" circularité (Cf. Carrion, 1984).
Quant à moi, je crois que c'est la dernière voie que prend Hart: le rapport entre la règle de reconnaissance et les autres règles du système, dont elle fournit les critères d'existence et d'identité, est en effet circulaire. Ce qui, en dernière analyse, détermine le contenu de la règle de reconnaissance est, tout simplement, l'accord dans les jugements proférés, d'un point de vue interne, par les membres de la communauté, tout comme, pour reprendre le mot de Wittgenstein, "ce que nous appelons "mesurer" est partiellement déterminé par la constance dans les résultats de la mesure" (Wittgenstein, 1953, $\S 242)$.

Que c'est bien une telle sorte de cercle vertueux qui est visé par Hart, c'est ce que je veux dégager de ses remarques concernant le mode d'existence de la règle de reconnaissance. Reprenons son exemple:

"Si la question est soulevée de savoir si une certaine règle proposée est juridiquement valable, il nous faut, pour répondre à cette question, employer un critère de validité fourni par une autre règle. Cette prétendue ordonnance du County Council d'Oxfordshire est-elle valide? Oui: parce qu'elle a été faite dans l'exercice des pouvoirs conférés, et en accord avec le procédé spécifié, par un décret du ministre de la santé". (Hart, 1961, 104)

Jusqu'ici, le décret fournit les critères par lesquels la validité de l'ordonnance est jugée - en stricte obéissance au principe qui veut que la validité d'une règle soit assurée par une autre règle (qu'on ne dérive pas un "doit" d'un "est"). Il se peut que l'on n'ait pas besoin d'aller plus loin, mais ce qui est important est le fait qu'on puisse le faire:

"Nous pouvons mettre en question la validité du décret ministériel, et déterminer sa validité en accord avec une loi conférant au Ministre le pouvoir de promulguer ces décrets. Finalement, quand la question de la validité de la loi a été suscitée et décidée par référence à la règle selon laquelle est loi ce que la Reine au Parlament promulgue, nous sommes arrivés à une halte dans les recherches sur la validité: car nous avons trouvé une règle qui, tout comme le décret 
et la loi intermédiaires, fournit des critères de détermination de la validité d'autres règles; mais elle est différente des autres en ce qu'il n'y a pas de règle qui fournisse des critères pour la détermination de la validité de cette règle-là". (Hart, 1961, 104; je souligne, PF)

C'est en arrivant à cette halte que, dans les théories normativistes, on bascule vers l'inconditionné, qu'il s'agisse d'une règle ou d'un ensemble de règles transcendantes (dans les doctrines classiques du droit naturel), ou d'une pure fiction méthodologique (comme la "Grundnorn" dans cette "philosophie du comme-si" qu'est, finalement, la théorie pure du droit).

Ce n'est pas le cas pour Hart, dont la perspective est, pour reprendre le mot de Raz, celle de la normativité sociale, par opposition à la normativité justifiée (Raz, 1979, $134)^{16}$; ce qui revient à dire que, littéralement, il ne peut y avoir, pour Hart, d'autre concept de validité que celui de la validité d'une règle à l'intérieur d'un systéme de règles. En d'autres termes, il ne peut pas y être question de la validité $d u$ système comme un tout ${ }^{17}$. A fortiori, il ne peut y être question de la validité de la règle de reconnaissance:

"Nous avons besoin du mot "validité", et normalement nous l'employons, pour répondre à des questions qui se posent à l'intérieur d'un système de règles où le statut d'une règle en tant que membre du système dépend de ce qu'elle s'accorde aux critères fournis par la règle de reconnaisance. Aucune question de ce type ne peut surgir en ce qui concerne la validité de la règle de reconnaissance qui fournit les critères; elle ne peut pas être valide ni invalide, mais tout au plus acceptée comme adéquate à cet emploi". (Hart, 1961, 105-106)

En accord avec cela, la question de l'existence de la règle de reconnaissance devient "une question de fait empirique" (Hart, 1961, 245). Qu'il y ait une telle règle, cela revient à dire qu'en fait les gens reconnaissent (identifient) les règles selon ce critère. L'accord dans les jugements de reconnaissance est, dans un sens très strict, tout ce dont il peut être question lorsqu'on s'interroge sur l'existence de la règle de reconnaissance. Comme l'écrit Hart:

"La plupart du temps la règle n'est pas énoncée, mais son existence s'exbibe dans la manière dont les règles particulières son identifiées, que ce soit par les tribunaux ou autres autorités, ou par des personnes privées, au moyen de ce critère". (Hart, 1961, 98)

Dans un certain sens, il serait donc correct de dire, à la manière de Kelsen, que la règle de reconnaissance est présupposée. L'idée de présupposition qui est à l'oeuvre ici est pourtant tout autre chose que celle d'une "condition logico-transcendentale" (comme le dit Kelsen) de l'attribution d'un sens normatif aux actes fondateurs de l'ordre juridique (Cf. Kelsen, 1960, 208-214). Dire que la règle de reconnaissance est présupposée est tout simplement dire que les règles du système sont identifiées d'une certaine façon, en accord avec des critères que l'on pourrait, si besoin était, rendre explicites:

"Exprimer ce fait simple en disant obscurément que sa validité est "supposée mais ne peut pas être démontrée" est la même chose que dire que nous supposons mais ne pouvons jamais démontrer que le mètre-étalon à Paris, qui est l'épreuve définitive de correction pour toute mesure en mètres, est lui-même correct". (Hart, 1961, 106)

Le mode d'existence de la règle de reconnaissance est donc celui d'une pratique (et il faudrait le dire: d'une pratique langagière) partagée: c'est le fait que les gens acceptent cette règle, certifié par l'usage qu'ils en font, en proférant des énoncés internes, qui en constitue l'existence, un point c'est tout.

Reconnaître l'existence d'une règle, cela relève de la maîtrise du langage, c'est-à-dire de l'usage que nous faisons des mots. Si j'ai souligné le fait qu'une même phrase peut être employée soit pour justifier, critiquer ou exiger des actions, soit pour décrire un état de choses (ce que j'ai appelé l'usage engagé et l'usage détaché d'une "proposition normative") c'était pour rappeler cette trivialité, à savoir que les mots n'ont pas de sens détachable de l'usage que nous en faisons.
L'absurdité (plutôt que la "fausseté") du cepticisme sur les règles devient évidente lorsqu'on se rend compte de ce qu'il est incapable de distinguer les deux types d'enoncés auxquels nous venons de faire allusion, et que c'est finalement la croyance (que j'appellerai volontiers platonique) à la détachabilité d'un "contenu propositionnel" qui serait encore de quelque manière au-dessus de l'emploi de la proposition, que soutient l'illusion d'un usage fondamental (dans le cas présent, l'usage cognitif) du langage, auquel on pourrait finalement reconduire tous les autres. Mais ce qui est peut-être plus important, c'est là aussi, je le crois, la racine de l'idée même d'une fonda tion, au sens philosophique, du politique.

A quoi il faut ajouter que là même où l'on fait un usage proprement cognitif du langage (en affirmant ou niant une existence ou un état de choses), c'est encore et toujours un acte qu'on accomplit - un acte duquel on peut toujours être appelé à rendre compte et raison, ce qui entraîne déjà le passage au discours pratique.

On peut disputer sur la règle de reconnaissance. Mais cela ne veut pas dire: disputer s'il y $a$ une règle de reconnaissance. Tou au plus, cela veut dire: disputer sur la règle de reconnaissance qu'il faut employer. E c'est souvent en cela que consiste une controverse politique.

La moralité à dégager de cette histoire est peut-être celle que Stanley Cavell, dans The Claim of Reason, exprimait par ces mots:

"Le dissens, ce n'est pas l'anéantissement du consensus, c'est un différend sur son contenu, un différend interne à lui sur la question de savoir si un arrangement présent lui est fidèle. L'alternative à parler pour soi-même politiquement, ce n'est pas: parler pour soi-même en privé (...) L'alternative, c'est ne rien avoir (politiquement) dire". (Cavell, 1979, 278)

Le corollaire de cette conclusion, cependant, devrait être l'acceptation, "désillusionnée" si l'on veut, du statut indépassablement problématique du jugement de reconnaissance: la règle de ce jugement n'a pas d'existence indépendante des "applica- tions" qui, littéralement, en déterminent le contenu.

Dans cettre perspective, l'idée de démocratie se laisse reconstruire comme étant fondée sur un refus principiel de l'idée même d'un jugement conclusif ${ }^{18}$ de reconnaissance, refus dont la contrepartie est l'institution d'un succédané interne au point de vue de l'extériorité radicale (la citoyenneté assurée au dissident), qui caractérise le pluralisme politique.

Maintenant vous pouvez naturellement opposer à tont ce que je viens de dire que, dès le début, j'ai supposé qu'une langue de choucas est tradusible et que, ce faisant, j'ai en fait joué avec des dés pipés.

A quoi il faut répondre qu'on est toujours libre d'imaginer que ces cris de choucas ne soient pas vraiment intelligibles. Mais, dans ce cas-là, on n'a en revanche aucune raison d'appeler cela un langage ${ }^{19}$.

\section{Notes}

${ }^{1}$ Renvoi obligatoire, à ce propos, à l'article de Stroud (1968), et à la discussion qui s'ensuivit. Cf. pour une révision récente (avec laquelle je suis d'accord pour l'essentiel), Förster, 1989.

${ }^{2} \mathrm{Ce}$ qui, vue l'interdefinibilité des opérateurs déontiques, comprend tout aussi des permissions et des prohibitions. Les règles "imposant des devoirs" sont distinguer de celles, "secondaires", qui portent sur la création, la suppression, l'application et, last but no least, sur la reconnaissance des premières. Cf, pour une excellente présentation générale, Bobbio, 1968.

3 Le programme est comparable à celui de l'elimina Le programme est comparable à clu' de lelimina tion des "termes théoriques" en faveur d un vocabulaire purement "observationnel", qui a hanté la philosophie des sciences naturelles aux annees heroiques du positi-
visme logique.

Extensionnellement équivalentes: elles ont les mêmes conditions de vérité.

5 Un plaidoyer, inspiré de Weber et de Wittgenstein, pour un abordage "herméneutique" des sciences sociales. Ce livre est cité, avec approbation, deux fois dan The Concept of Law (Cf. Hart 1961, 242, 249). Il me semble, pourtant, que les racines de la perspective "herméneutique" de Hart sont à rechercher plutôt du côté de la "philosophie du langage ordinaire" à la manière d'Oxford après-guerre, et notamment dans la théorie des actes de langage (à la naissance de laquelle Hart a joú un rôle important dans son travail avec Austin) que dans les développements se réclamant de Wittgens- 
tein dans la méthodologie des sciences sociales (tels ceux de Winch ou, un peu plus tard, de Von Wright). ${ }^{6}$ Je répète encore une fois, pour moi-même, le premier mouvement de la sonate "Waldstein". Sous la description "Jouer du piano chez moi", j’ai réalisé une action permise (par les normes du voisinage, disons). Sous la description "Faire du bruit dans le bâtiment après 22 h", j'ai réalisé une action défendue. Ne demandez pas, s'il vous plaît, si c'est bien la même action qu'on décri dans les deux cas (à mon avis, quand même: oui). J'avoue, avec un peu d'honte, avoir été pendant un temps séduit par le mirage d'une "réfutation du scep ticisme" dont le noyau serait l'idée que l'individuation d'une action est toujours faite "sous une description", ce que, imprudemment, j'étais enclin à prendre comme équivalant à dire qu'une action est toujours un "fai institutionnel", au sens de Searle (1969). (Et, bien sûr, des actions le sont souvent, mais pas du tout nécessairement). J'ai été éveillé de mon sommeil dogmatique par l'article admirable d'Elizabeth Anscombe (1979), d'où je cède à la tentation d'extraire cette remarque méthodologique générale: "Etant donné un argumen sur l'individuation ou l'identité d'un événement ou d'une action, nous pouvons souvent construire un argument parallèle sur l'individuation de choses concrètes, qui serait sensé si l'argument sur les événements et les actions l'etait, mais qui est manifestement absurde. Là où un argument sur des événements ou des actions peut être éprouvé de cette façon, il devrait 'être; nous serons souvent en état de révéler ainsi du non-sens latent" (Anscombe, 1979, 213).

${ }^{7}$ On peut lire la théorie kelsenienne des "domaines $\mathrm{de}$ validité" de la règle de droit comme une théorie sémantique de l'observance. C'est l'idée (disputable) de Vernengo (1964). Cf. aussi Eisenmann, 1964

${ }^{8}$ C'est bien la suggestion de MacCormick, pour qui 1 point de vue herméneutique de $\mathrm{Hart}$ débouche forcément sur une perspective intentionnaliste (MacCormick, 1978, 284).

9 L'acceptation d'une règle n'est pas à confondre avec l'approbation, c'est-à-dire avec un jugement sur la règle, à savoir, qu'il s'agit d'une bonne règle. Comme le remarque Bobbio: "L'acceptation est distincte de simple conformité, c'est-à-dire de l'obéissance ou observance mécanique, habituelle, de la norme. Elle est distincte, aussi bien, de l'approbation, c'est-à-dire de l'attribution à la norme d'une valeur éthique, de conviction qu'il s'agit d'une bonne norme: les jues, lorsqu'ils font usage d'une norme, comme critère jugement du comportement d'autrui et comme justifcation pour metre en mouvenent'apparat de justifion, accertat la norme, encore qu'on ne dise pas par lì qu'ils lapprouvent, qu'ils la tiennent pour just" $(1966,143)$. Cf pour une vue differente, Com ja 1971.

${ }^{10}$ Comme le remarquent Baker et Hacker, "des règles que personne ne cite pour expliquer ce qu'il est correct de faire, auxquelles personne ne se réere pour justifier ce qu'il a fait ou pour critiquer des autres qui ont ag incorrectement (...) sont en fait des objets douteux. 1 n'est pas étonnant que le scepticisme sur les règles semble une préoccupation philosophique profonde et importante!" (1984, IX)

${ }^{11}$ C'est pourquoi on est contraint d'introduire, dans la notation, ce que Wittgenstein appelait les "crochets" (Haken): les guillemets, par exemple.

${ }^{12}$ La conclusion de l'argument donné en exemple par Searle dans sa fameuse "preuve" de la permissibilité de dériver un "doit" d'un "est" n'est "impliquée" par les prémisses que si elle est prise pour un énoncé détaché, au sens de Raz. Tout l'intérêt de la trouvaille de Searle dépend, précisément, de ce qu'aucune forme propositionnelle spéciale ne caractérise les deux usages de la proposition (Cf. Searle, 1969, 177-188).

${ }^{13} \mathrm{Ce}$ que le discours du dissident partage avec celui du positivisme juridique (reconnaittre les règles sans les accepter), voilà ce que rend "indésirable" ce dernier (Cf. Kelsen, 1960, 10)

${ }^{14} \mathrm{~A}$ mon avis, la théorie kantienne de la preuve philosophique, telle que la presente la "Doctrine transcendentale de la Méthode" dans la Critique de la Raison Pure, demeure, de nos jours, l'effort le plus remarquable d'un traitement systématique de la question. L'examen de cette théorie, aussi bien que du rapport problématique (pour dire le moins) entre cette théorie et la pratique de Kant (dans les preuves de l'Esthétique et de l'Analytique Transcendentales) appartient au sujet d'un ouvrage que je prépare à présent sur le "scandale de la philosophie" (Cf. Kant, 1781, B XXXIX).

${ }^{15} \mathrm{Si}$ j'avais l'occasion, je tâcherai de caractériser d'une façon précise cette stratégie, en faisant appel à la notion, introduite par Nelson Goodman et reprise et développée par John Rawls, d'équilibre réflexif, c'est-àdire de "l'accord réciproque entre des principes et des jugements considérés" (Rawls, 1971, 20; je souligne, PF; Cf. Goodman, 1954, 62-66; Rawls, 1971, 20-21, 48-51). 16 "Deux conceptions de la normativité du droit sont courantes. Je les appellerai normativité justifiée et normativité sociale. Selon la première vue, des étalons juridiques de comportement sont des règles seulement si, et pour autant que, ils sont justifiés. Ils peuvent être justifiés par des raisons objectivement valides. Ils peuvent être perçus intuitivement comme obligatoires, ou bien ils peuvent être acceptés comme obustifiés en raison d'un engagement personnel. Selon l'autre vue des átadons de des norm, des nomes abstration faice de son merite. lls sont des normes sociales pour autant qu'lls sont socialement consideres conme des etalons obligatoires, et que la societé en question exerce une pression sur les gens auxquels les etalons $s$ appliquent pour qu'ils $s^{\prime} y$ conforment. Les thericiens du droit naturel typiquement souscrivent à la première vue, les positivistes usuellement maintiennent la deuxième" (Raz, 1979, 134). Lobscurite de la theorie kelsenienne de la "norme fondamentale", et les controverses auxquelles cette théorie a donné lieu, tiennent, selon Raz, pour une bonne partie, au fait (peu reconnu) que Kelsen, n'em- ploie d'autre concept de normativité que celui de la normativité justifiée (Cf. Raz, 1979, 134-143).

$17 \mathrm{Ce}$ qui, bien sûr, n'exlut pas qu'il puisse y être question de sa valeur morale: Cf. Hart, 1958.

${ }^{18}$ Au sens de: "chose jugée".

19 A Saint-Cloud, M. Jean-Marie Vincent proposait d'appeler "réalisme linguistique" Pinterprétation que j'ai présentée de la philosophie du droit de Hart; voulait par là signaler ce que cette interprétation partage encore avec le "realisme" sceptique auquel el s'opposait ostensiblement: à savoir, la suspension du jugement en ce qui concerne les règles, s'il y en a, do l'existence ne soit pas une fonction de source, c'est-d dire de reconnaissance, au sens ou jai employe ce mo Cette limitation est, pourtant, ici comme ailleurs, prix à payer pour "battre le sceptique sur son propre terrain": tout comme, si l'on veut, l acceptation "transcendantale" du scepticisme épistémique, chez Kant (on ne peut connaitre que des representations, pas les cho ses en soi) est le prix à payer pour se débarrasser du scepticisme au niveau "empirique". Que la manoeuvre coûte, tout compte fait, plutôt cher, je l'accorderais volontiers. Mais à condition que l'on voie bien ce qu'un tel bilan met en cause: à savoir l'idee même que la philosophie ait affaire à des preuves et des refutations. Pour ce qui est de la "vraie" normativité (celle, bien sûr, de l'inconditionné), quoi qu'il en soit, il se peut que la raison renvienne finalement à Wittgenstein, qui remarquait, dans ses cahiers (le 15 de novembre 1929): "Tu ne peux pas mener les gens vers le bien; tout au plus tu peux les mener quelque part. Le bien demeure au dehors de l'espace des faits" (Wittgenstein, 1967, 117).

\section{Références}

Anscombe (G.E.M.)

1979 "Under a Description" in Metaphysics and the Phi

losophy of Mind (The Collected Philosophical Papers of G.E.M. Anscombe, Vol. II) (Minneapolis, University of Minnesota Press)

Baker (G.P.) \& Hacker (P.M.S.)

1984 Scepticism, Rules and Language (Oxford, Basil Blackwell).

Bobbio (N.)

1966 "Considerazioni in Margine" in Guastini (1980) pp. 135-144.

968 "Norme Primarie e Norme Secondarie" in Studi per una Teoria Generale del Diritto (Torino, G. Giappichelli Editore, 1970), pp. 175-197.

Bulygin (E.)

1976 "Sobre la Regla de Reconocimiento" in (plusieurs auteurs) Derecho, Filosofia y Lenguaje (Buenos Aires, Astrea).

Carrion (R.)
1984 "A Questão da Justificação das Regras" in Aquiles no 1 (Porto Alegre, Mercado Aberto/UFGRS, 1984), pp. 9-11.

Catania (A.)

1971 "L'accetazione nel Pensiero di Herbert L.A. Hart", Rivista Internazionale di Filosofia del Diritto, 1971, pp. 261-279.

\section{Eisenmann (Ch.)}

1964 "A propos de la Théorie Kelsenienne des Domajnes de Validité" in Engel (1964).

Engel (S.) (ed.)

964 Law State and International Legal Order - Essays in Honour of Hans Kelsen (Knoxville, The University of Tennessee Press).

Förster (E.)

1989 "How are Transcendental Arguments Possible?" in Schaper, E. \& Vossenkuhl, W. (eds.) Reading Kant (Oxford, Basil Blackwell)

Goodman (N.)

1954 Fact, Fiction and Forecast, quatrième édition (London and Cambridge, Mass., Harvard University Press, 1973)

Guastini (R.) (ed.)

1980 Problemi di Teoria del Diritto (Bologna, Il Mulino) Hart (H.L.A.)

1953 Definition and Theory in Jurisprudence (Oxford, Clarendon Press).

1958 "Positivism and the Separation of Law and Mo rals" in Harvard Law Review, Vol. 71, n. 4, pp 593-629.

1959 "Scandinavian Realism", reproduit in Hart (1983), pp. 161-169.

1961 The Concept of Law (Oxford, Clarendon Press).

1963 "Kelsen Visited", reproduit in Hart (1983), pp 286-308.

1983 Essays in Jurisprudence and Philosophy (Oxford, Clarendon Press)

Kafka (F.)

1917 "Une Vieille Page" (Un Vieux Parchemin), trad. A Vialatte, in OEuvres Completes, Vol. II (Paris, Gallimard, 1980).

Kant (I.)

81 Critique de la Raison Pure, trad. A. Delamarre et F. Marty in OEuvres Pbilosophiques, Vol. I (Paris, Gallimard, 1980)

1960 Teoria Pura del Derecho, trad. R. Vernengo (Mexico, UNAM, 1979).

MacCormick (N.)

1978 Legal Reasoning and Legal Theory (Oxford, Clarendon Press).

Olivecrona (K.) 
1971 Law as Fact, second edition (London, Stevens \& Sons).

Rawls (J.)

1971 A Theory of Justice (Oxford University Press).

$\operatorname{Raz}(\mathrm{J}$.

1979 The Autbority of Law (Oxford, Clarendon Press).

Ross (A.)

1962 "Su Hart", trad. R. Guastini, in Guastini (1980),

pp. $355-360$.

Searle (J.R.)

1969 Speech Acts (Cambridge, Cambridge University Press).

Sextus Empiricus

1948 Les Esquisses Pyrrhoniennes ou Hypotyposes, trad. G. Goron, in OEuvres Choisies de Sextus Empiricus (Paris, Aubier/Montaigne).

Stroud (B.)

1968 "Transcendental Arguments", in The Journal of Pbilosophy, Vol. LXV, no 9, pp. 241-256.

Swift (J.)

1726 Gulliver's Travels, ed. Ch. Lloyd (London, Longman, 1971)

Vernengo (R.)

1964 "About Some Formation Rules for Legal Languages", in Engel (1964).

Winch (P.)

1958 The Idea of a Social Science (London, Routledge \& Kegan Paul).

Wittgenstein (L.)

1953 Philosophische Untersuchungen, ed. G.E.M. Anscombe, G.H. Von Wright \& R. Rhees, in Werkausgabe, Band 1 (Frankfurt, Suhrkamp, 1989).

1967 Ludwig Wittgenstein und der Wiener Kreis, Schriffen, Band 3 (Frankfurt, Suhrkamp, 1980).

\section{Basic Problems of Harmonizing Tax Law in the European Communities}

Prof. Dr. Gerhard Laule

Professor, University of Saarbrucken, Member International

Fiscal Association, Board Member of the German IFA-Branch,

International Law Association, Gesellschaft für Finanzwirtschaft in der

Unternehmensführung; International Institute of Public Finance.

TABLE OF CONTENTS

1. Introduction, Review of EEC tax developments; 2. Excise Duties and VAT; 2.1 Excise Duties; 2.2 Value-added Tax; 3. Harmonization of Direct Taxes; 4. Trade Tax and Capital Tax; 4.1 The Trade Tax; 4.2 The Capital Tax; 4.3 Arguments against the discriminating, Trade and Capital Taxes; 5. Income Tax on Individuals; 5.1 Liability to Individual, Income Tax on Trade or Business; 5.2 Tax Scales; 6. Corporate Income Tax; 6.1 The Corporate Income Tax System; 6.2 Liability to Corporation Tax; 6.3 Integrated Companies; 6.4 The Tax Rates; 6.5 Mergers and Acquisitions; 7. Taxable Income; 7.1 Capital Gains; 7.2 Deduction of Losses; 7.3 Trends of the EEC for the Determination of Corporate Income; 8. European Communities Taxation of Corporation; 8.1 Corporation Tax Systems; 8.2 Tax Rates; 8.3 Trends; 9. Summary.

1. Introduction - Review of EEC tax developments

The EEC has recently established principals on a European tax system.

Indirect taxation, VAT as well as excise duties, are subject to political agreements on the harmonization in the EEC after 1992.

Customs have already been uniform since 25 years.

The Commission's new approach on direct tax developments seeks the mutual coordination and approximation of national tax policies by the Member States'.

The Treaty of Rome - the "Treaty" - explicitly deals with indirect taxes, outlawing not only customs duties and export subsidies, which are an obstacle to the free novement of persons, goods, services and capital the four freedoms - within the EEC, but other indirect tax measures, wich would have equivalent effects (Art. 95 to 99 of the Treaty).

The legal basis on harmonization or only coordination of enterprise taxation in the EEC is rather weak. Unlike the indirect taxes - which include in particular the value added tax -, none of the articles provides specifically for a harmonization of direct taxation ${ }^{2}$.
The Commission decided to give priority to measures required to eliminate or reduce obstacles to cross-border activities by 1993, an approach, which accords with the Principle of Subsidiarity; policies should be forged at the most local level which is feasible. The feasibility of implemeting a policy at the local level is closely related to the magnitude of any interjurisdictional spillover effect associated with it ${ }^{3}$.

\section{Excise duties and VAT}

The Coordination of indirect tax systems is provided for by rather precise requirements of the Treaty.

The EEC and the Member States have passed resolutions and agreements on abolishing frontier controls on goods subject to excise duties or VAT.

\subsection{Excise duties}

In September 1990, the EEC Commission introduced proposals on excise duties which are designed to introduce a system of interlinked bonded warehouses and provide a harmonized definition of how excise duties should apply to alcohol, tobacco and mineral oils ${ }^{4}$. 